\title{
Dynamics of phase-locked semiconductor laser arrays
}

\author{
S. S. Wang and H. G. Wintul \\ Deparment of Electrical Engineering and Computer Science, Uniwersity of Michigan, \\ Ann Arbor, Michigan 48109
}

(Received 7 December 1987; accepted for publication 21 March 1988)

\begin{abstract}
Time-dependent coupled mode theory is used to investigate the stability of phase-locked semiconductor laser arrays. The output of individual array elements is dynamically unstable and exhibits large amplitude chaotic pulsations. The total output initially exhibits damped relaxation oscillations and then settles down to a quasi-steady state characterized by small amplitude fuctuations. The theory predicts both the pulsation frequency and the phase lock-in time of the array.
\end{abstract}

Phase-locked semiconductor laser arrays have been demonstrated as sources that can produce high output power in a spatially coherent beam.' The spatial mode patterns observed ${ }^{2}$ under steady-state conditions are predicted rather well by coupled mode theory which leads to a description of the feld profies in terms of array modes or supermodes. ${ }^{3,4}$ While the steady-state behavior of phase-locked arrays has been the subject of numerous investigations, there is relatively lithle data avalable on the transient response of these arrays. Recent streak camera measurements have shown that the output of individual elements of the array exhibits irregular, ancamped spiking behavior on a $100 \mathrm{ps}$ time scale. The temporal stability of array operation is thus an issue that merits further investigation.

In this letter, we present a theoretical analysis of the temporal behavior of phase-locked semiconductor laser arrays. The analysis is based on semiclassical laser theory ${ }^{6}$ modified to include terms that describe evanescent wave coupling between adjacent elements of the array. The numerical results are in excellent qualitative agreement with the available experimental data on the dyramic characteristics of laser arrays. ${ }^{5}$ In particular, the theory shows that the output of the individual array elements is dynamically unstable and exhibits chaotic pulsations. The theory also yields the period of the pulsations and the establishment time of phase locking, both of which are inversely proportional to the coupling coeficient between array elements.

An array of $N$ coupled hasers is considered. When isolated from its neighbors, each laser supports a single transverse and longitudinal mode assumed same for all the lasers. The temporal evolution of the carrier density $\left(N_{i}\right)$ and of the amplitude $\left(E_{i}\right)$ and phase $\left(\phi_{i}\right)$ of the clectric field in the ith channel of the array is described by

$$
\begin{aligned}
\frac{d E_{i}}{d i}= & \frac{1}{2}\left(\Gamma G\left(N_{i}\right)-\frac{1}{\tau_{p}}\right) E_{i}-\frac{\kappa c}{n} \\
& \times\left[E_{i+1} \sin \left(\phi_{i+1}-\phi_{i}\right)+E_{i-1} \sin \left(\phi_{i-1}-\phi_{i}\right)\right],
\end{aligned}
$$

$$
\begin{aligned}
\frac{d \phi_{i}}{d t}= & \omega\left(N_{\mathrm{th}}\right)-\omega\left(N_{i}\right)+\frac{\kappa c}{n}\left(\frac{E_{i+1}}{E_{i}} \cos \left(\phi_{i+1}-\phi_{i}\right)\right. \\
& \left.+\frac{E_{i-1}}{E_{i}} \cos \left(\phi_{i-1}-\phi_{i}\right)\right),
\end{aligned}
$$

$\frac{d N_{i}}{d t}=\frac{J}{e d}-\frac{N_{i}}{\tau_{s}}-\Gamma G\left(N_{i}\right)\left|E_{i}\right|^{2}$

Here the field amplitude is normalized sach that $\left|E_{i}\right|^{2}$ gives the photon density in the ith channel. The constant parameters in the above equations are the coupling coefficient $\kappa$, mode coninnement factor $\Gamma$, the photon lifetime $\tau_{p}$, the carrier lifetime $\tau_{s}$, the injection current density $J_{s}$, the widh $d$ of the active region, and the electronic charge $e$. The gain $G\left(N_{i}\right)$ and the laser frequency $\omega\left(N_{i}\right)$ depend on the carrier density through

$$
\begin{aligned}
& G\left(N_{i}\right)=G\left(N_{\mathrm{th}}\right)+\left(\frac{\partial G}{\partial N_{i}}\right)\left(N_{i}-N_{\mathrm{th}}\right), \\
& \omega\left(N_{i}\right)=\omega\left(N_{\mathrm{th}}\right)+h\left(N_{i}-N_{\mathrm{th}}\right),
\end{aligned}
$$

where $N_{t h}$ is the threshold carrier density, $h=d \omega / d N_{i}=(\omega / n) d n / d N_{i}$, and the derivatives are evaluated at threshold. In writing down these equations, we have neglected a small term representing spontaneous emission into the lasing mode. Its inclusion in our simulations produced negligible effects.

\begin{tabular}{|c|c|c|c|}
\hline Parameter & Symbol & Numerica? value & Unit \\
\hline Current density & $d$ & $3 \times 10^{6}$ & $A / \mathrm{rn}^{2}$ \\
\hline Active layer thickness & $d$ & 300 & $\AA$ \\
\hline Carner lifetime & $\tau_{\mathrm{s}}$ & 2 & ns \\
\hline Photon lifetime & $\tau_{p}$ & 1.6 & ps \\
\hline Fhreshold carrier density & $N_{\mathrm{th}}$ & $1.0 \times 10^{24}$ & $m^{-3}$ \\
\hline Refractive incex & $n$ & 3.6 & $\cdots$ \\
\hline Coupling cocficient & $\kappa$ & 317 & $\mathrm{~m}^{-1}$ \\
\hline Gain at threshold & $G\left(N_{t h}\right)$ & $1.24 \times 10^{12}$ & $s^{-1}$ \\
\hline $\begin{array}{l}\text { Differential gain } \\
\text { Rate of change of resonant }\end{array}$ & $\partial G / \partial N$ & $0.856 \times 10^{-6.3}$ & $\mathrm{~m}^{5} / \mathrm{s}$ \\
\hline $\begin{array}{l}\text { frequency } \\
\text { Fraction of power in active }\end{array}$ & $h$ & $2.14 \times 10^{12}$ & $\mathrm{~m}^{3} / \mathrm{s}$ \\
\hline region & $\Gamma$ & 0.46 & $\cdots$ \\
\hline
\end{tabular}

It should be noted that even though the isolated laser stripes all have the same single frequency, once they are coupled the oscillating frequency splits into $N$ different values, one for each supermode. In the absence of nonlinearity, these

TABLE 1. Parameters ased in the numerical simulations. 
supermodes would simply beat against one another leading to a periodic or quasiperiodic output in time. The fundamental beat frequency is given approximately by $(2 / \pi)(\mathrm{kc} / \mathrm{n})$. It should also be noted that the amplitude and phase equations (1) and (2) are identical to the equations that describe FM laser oscillation and active FM node locking. ${ }^{7}$ Similar multimode behavior is thus expected from the coupled array.

The time-dependent coupled mode equations have been soived numerically by means of a program geared for stiff differential equations. A ten-element array was simulated, which required the solution of 30 coupled nonlinear differential equations. The values of the parameters used are given in Table $I$ and the numerical results for a step imput current are presented in Figs. 1-3.

Figure 1 shows the single-shot intensity of an individual array element as measured in the near field. The trace shows strong relaxation oscllations initially and then undanped spiking behavior for the duration of the injection current pulse. The pulsations are chaotic and show no sign of damping out even after times as long as $50 \mathrm{~ns}$, which is 20 times the carrier lifetime. These results agree with the experimental observations of Ellot et al. who made single-shot streak camera measurements of the pulsed ontput of individual ar. ray elements.

The total output of the array, also measured in the near feld, is shown in Fig. 2. Unlike the output of the individual elements, the tota intensity exhibits relaxation osciliations which damp out to a quasi-steady state characterized by a much reduced level of fluctuations.

Figure 3 shows the output of an individual array element averaged over ten shots. The fields were given random initial phases which varied from shot to shot. It is seen that the average output intensity from individual elements exhibits reduced fuctuations. This resut also agrees with the experimental observations.

It is clear from these results that even though the total output of a phase-locked laser array may be steady in time, the individua strine intensities can continue to pulsate in an irregular manner. Such behavior is generic to multimode lasers and is due, in this case, fo competition between array supermodes.

The spiking behavio in the first 10 ns is simply the col-

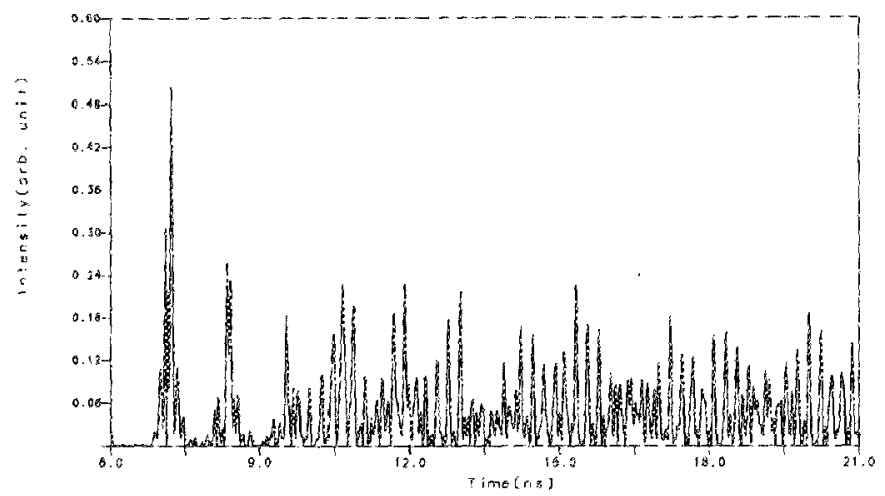

FIG. 1. Single-shot intensity of light emitted by ars individuul laser in the array (stripe Ro. 1).

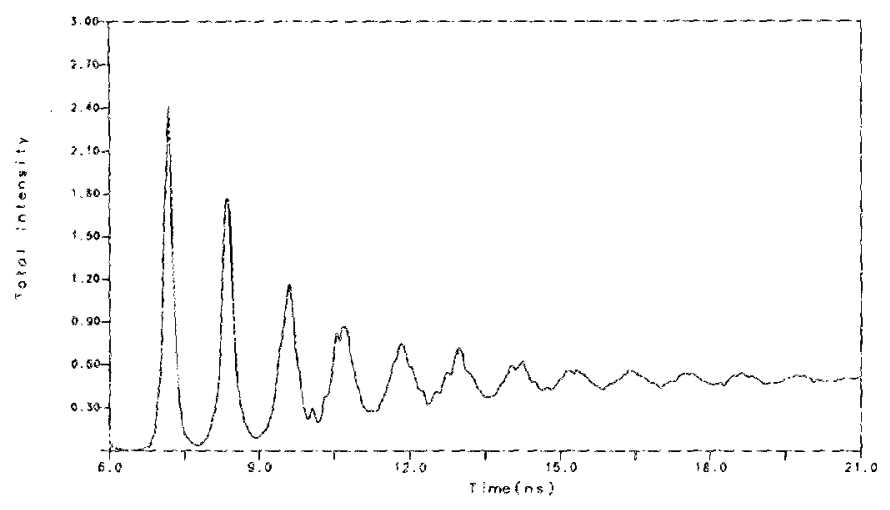

FIC. 2. Time evolution of the total intensity (near field) emited in a single pulse by a ten-element array.

lective relaxation oscillations of the semiconductor laser ensemble. The characteristic frequency of this oscillation is governed by the rate of energy transfer between electrons and photons in a single laser stripe. This frequency is proportional to the square root of the driving current, inversely proportional to the geometric thean of the photon and carrier lifetimes, and independent of the coupling berween laser stripes. After the initial relaxation oscillations, the individual array elements enter anew oscillatory regime characterized by the rate of energy transfer between elements. The frequency of this ascillation is proportional to the coupling coefficient $\mathrm{kc} / \mathrm{n}$. For a coupling constant of $k=100 \mathrm{~m}^{-\mathrm{l}}$, the pulsation frequency is in the $10 \mathrm{CH} z$ range, which is in agreenent with the experimental value obtained by Elliot of al. Measurement of the pulsation period may provide a means of determining the coupling constant $k$.

In the absence of frequency-pulling effects (i.e, $h=0$ ), the coupling pulsations would be periodic, or at worst, quastperiodic. The dependence of the resonance frequency on the excitation level within each laser stripe $(h \neq 0)$ causes the array to behave as a set of coupled nonlinear oscillators. For moderate values of the coupling strength, the pulsations are chaotic. A fast detector that can resolve the output of individual array members will thus measure behavior akin to mode partition noise: individual modes show random behavior in time while the total intensity is steady. The difference

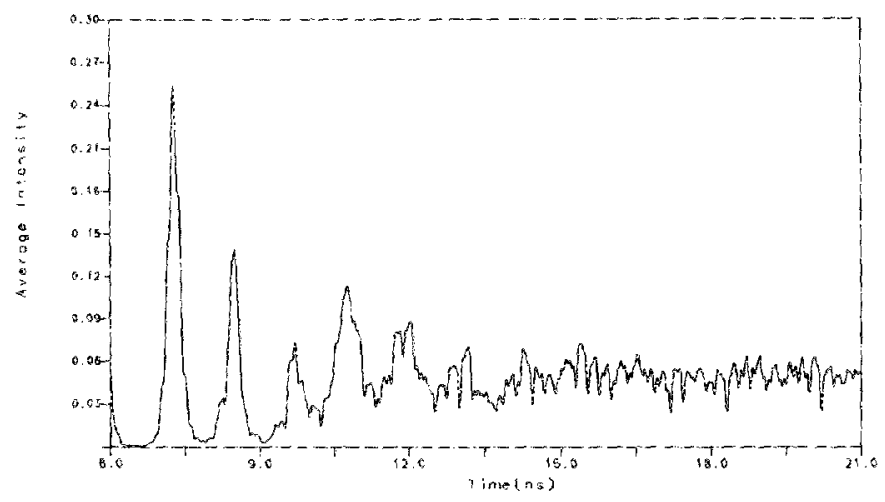

FIG. 3. Ten-puise average of the intensity cmitted by a single element in the array (stripe No. 1). 
here however, is that while mode partition noise arises from stochastic fuctuations, the behavior observed in this simulation is completely deterministic.

An important question concerning phase-iocked arrays is how long it takes for phase locking to be established after the initiation of lasing. That question can be answered by following procedures similar to Adler's analysis of injection locking. An approximate solution of the phase equation (2) shows that, to first order, locking is established with a time $(\mathrm{kc} / \mathrm{n})^{-1}$ following the initiation of lasing. Details of these calculations and of the evolution of the far-field patterns will be presented in a future publication.

The results presented here have important implications for the use of semiconductor laser arrays in applications where temporal stability is important. Laser arrays are intrinsically unstable. The dynamic instabilty seen here will adversely affect the modulation behavior of these arrays. On a more fundamental note, laser arrays offer the possibility for direct observation of spatio-temporal chaos in a system of coupled nonlinear osciliators.

This work was supported by the National Science Foundation through a Presidential Young Investigator Award to H. G. Winiul.

'For a recent review, see D. Botez and D. E. Ackley, IEEE Circuits Devices Mag. 2, 8 (1986).

27. I. Paoli, W. Streifer, and R. D. Burnham, Appl. Phys. Lett. 45, 217 $(1984)$.

${ }^{3}$ I. K. Butler, D. E. Ackley, and D. Botez, Appl Phys. Lett. 44, 293 (1984). ${ }^{4}$ E. Kapon, J. Katz, and A. Yariv, Opt. Lett. 10, 125 (1984).

${ }^{5}$ R. A. Elliot, R. K. Der reez, T. L. Paoli, R. D. Burmham, and W. Streifer, IEEE J. Quantum Llectron. QE-21, 598 (1985).

Gor a similar appronch to injection locking in semiconductor lasers, see $R$. Iang, IEEE \&. Quantum Electron. QE-18, 976 (1982).

7S. E. Harris and O. P. MeDufi, IEEE J. Quantum Electron, QE-1, 245 (1965).

"For an observation of deterministic chaos in a single stripe laser, see H. C. Winful, Y. C. Chen, and J. M. Liu, Appl. Plys. Lett. 48, 616 (1985). 